\title{
TRANSDISCIPLINARIEDAD, TRANSVERSALIDAD Y MODELOS DE FORMACIÓN ALTERNATIVOS
}

\author{
TRANSDISCIPLINARITY, TRANSVERSALITY AND \\ ALTERNATIVE TRAINING MODELS
}

\author{
Deicy Correa Mosquera* \\ Camila María Carlachiani**
}

Recibido: Julio 29, 2021

Aceptado: Octubre 22, 2021

\begin{abstract}
* Autora Principal. Magíster en Educación Superior de la Universidad Santiago de Cali- Colombia. Docente investigadora de la Universidad Autónoma de Chihuahua (México).

melocorrea06@hotmail.com 0000-0003-3904-1419

** Coautora. Magíster en Educación de la Universidad Nacional de La Plata de Argentina. Profesora en Ciencias de la Educación de la Universidad Nacional de Rosario (Argentina).

camilacarlachiani@gmail. com

0000-0002-4649-1541
\end{abstract}

Cómo citar este artículo: Correa, M. D. \& Carlachiani, C. M. (2021). Transdisciplinariedad, transversalidad y modelos de formación alternativos. Revista PACA 11, pp. 175196.
Resumen: Este documento tiene como propósito presentar algunos planteamientos sobre los conceptos de transdisciplinariedad y de transversalidad, los cuales pueden considerarse principios subyacentes a la construcción de modelos de formación orientados a la producción de nuevas prácticas pedagógicas en la educación superior que trasciendan las actuales maneras de enseñar, las cuales generalmente están basadas en currículos agregados (Bernstein, 1977).

Para tal efecto, en primer lugar se hace referencia a las relaciones entre transdisciplinariedad y transversalidad. En segundo lugar, se avanza en la conceptualización de lo que se entiende por modelo. En tercer lugar, se presentan algunas consideraciones sobre lo que configura un modelo de formación transversal para la educación superior. Finalmente, se elaboran algunas conclusiones y recomendaciones en relación con la importancia que tienen el fomentar el pensamiento transversal en los procesos de formación. Estos puntos son complejos, dada la diversidad de enfoques que hay sobre transdisciplinariedad, modelo, modelo de formación y modelo de formación transversal, y sobre el tratamiento aislado de estos conceptos que se han vuelto relevantes en la educación superior, especialmente en la universidad. De allí el interés en dar un abordaje sistémico acerca de los mismos, procurando establecer sus relaciones verticales y horizontales. 
Palabras clave: transdisciplinariedad, transversalidad, modelo, modelo de formación.

\begin{abstract}
The purpose of this paper is to present some approaches to the concepts of transdisciplinarity and transversality, which can be considered underlying principles of the construction of training models oriented to the production of new pedagogical practices in higher education that transcend current ways of teaching, which are generally based on aggregate curricula (Bernstein, 1977).
\end{abstract}

For this purpose, in the first place, reference is made to the relationships between transdisciplinarity and transversality. Second, progress is being made in the conceptualization of what is understood by a model. Third, some considerations are presented about what makes up a transversal training model. Finally, some conclusions and recommendations are drawn up in relation to the importance of promoting transversal thinking in training processes. These points are complex given the diversity of approaches that exist on transdisciplinarity, model, training model and transversal training model and on the isolated treatment of these concepts that have become relevant to higher education, especially for the universitiy,. Hence, the interest in giving a systemic approach to them, trying to establish their vertical and horizontal relationships.

$176 \quad$ Keywords: transversality, model, training model.

\title{
Introducción
}

Este documento tiene como propósito presentar algunos planteamientos sobre los conceptos de transdisciplinariedad y de transversalidad. El hecho que ellos hagan parte de las transformaciones sociopolíticas, económicas y culturales del presente siglo muestran que hoy no se producen cambios aislados, ni siquiera conceptuales y que la pluralidad y la integración son principios que definen no solo los cambios en la producción de conocimiento sino, también, en su reproducción vía educación. Desde esta perspectiva, la construcción de modelos de formación orientados a la producción de nuevas prácticas pedagógicas en la educación superior ha comenzado a inspirarse, así sea retóricamente, en la transdisciplinariedad y la transversalidad, conceptos que trascienden las actuales maneras de enseñar, las cuales generalmente están basadas en currículos agregados que representan la insularidad del conocimiento, incompatible con los signos del siglo XXI (Bernstein, 1977).

Con el propósito de fundamentar la relación entre transdisciplinariedad, transversalidad, modelos de formación y currículo, en primer lugar, se hace 
referencia a las relaciones entre transdisciplinariedad y transversalidad. En segundo lugar, se avanza en la conceptualización de lo que se entiende por modelo. En tercer lugar, se presentan algunas consideraciones sobre lo que configura un modelo de formación transversal para la educación superior y sus expresiones curriculares. Finalmente, se elaboran algunas conclusiones y recomendaciones en relación con la importancia que tienen el fomentar el pensamiento transversal en los procesos de formación. Estos puntos son complejos dada la diversidad de enfoques que hay sobre transdisciplinariedad, modelo, modelo de formación y modelo de formación transversal y sus expresiones curriculares, y sobre el tratamiento aislado de estos conceptos que se han vuelto relevantes en la educación superior, especialmente en la universidad. De allí el interés en dar un abordaje sistémico acerca de naturaleza, procurando establecer sus relaciones verticales y horizontales.

\section{Sobre la transdisciplinariedad y su relación con la transversalidad}

El concepto de transversalidad está directamente asociado a la existencia de los campos plurales que mantienen relaciones de unidad, diferencia e interdependencia. En consecuencia, no podemos pensarlo al margen de estas relaciones ni de sus cambios, independientemente de que, por ejemplo, los campos disciplinarios hayan sido considerados territorios aislados, separados, amurallados. La clasificación rígida entre las disciplinas es, quizás, el mayor obstáculo para la comprensión y ejercicio de la transdisciplinariedad. La rigidez del aislamiento disciplinario ha inhibido la construcción de conocimientos integrados que representen la totalidad, y la unidad de las disciplinas. Al referirse a la disciplinariedad, Dogan (2003) plantea que:

En todas las universidades, la enseñanza, el nombramiento y las carreras de los docentes, el examen de su labor por colegas de igual categoría se ajusta a las fronteras disciplinarias, (...). Por el mero hecho de existir oficialmente, una disciplina tiene muchos intereses profesionales que defender. Cada disciplina defiende celosamente su soberanía territorial. (p. 2).

Este planteamiento aún subsiste en la universidad, la cual mantiene sus archipiélagos tanto físicos como símbolos como expresiones de la territorialidad disciplinaria de la que habla Becher (2001). Por esto es conocido que, aún en el siglo XXI, las fronteras disciplinarias se mantienen. 
La universidad preserva celosamente los campos disciplinarios, que se materializan en una diversidad de espacios físicos fragmentados y divididos. Por una parte, tenemos las unidades académicas (departamentos, escuelas, centros, etc.) que agrupan a los profesores alrededor de una disciplina, profesión u objeto de investigación. Por la otra, vemos que la unidad clásica de organizar los currículos son las asignaturas. Es difícil encontrar un programa que no esté organizado en asignaturas. Estas representan la compartimentación de las disciplinas.

En la misma dirección, Edgar Morin (1990, p. 6) en su libro Lossiete saberes necesarios para la educación del futuro, critica la carencia de articulación entre los saberes que reproducen los conocimientos territorializados de las disciplinas, y las realidades o problemas contextuales que son vez más transversales, multidimensionales y globales. La mirada disciplinaria en la cual hemos forjado nuestra identidad epistémica ha constituido en nuestras conciencias formas de ver unilaterales, fragmentadas, del conocimiento, del mundo y de sus problemas. De allí que sea difícil pensar más allá de lo que legítimamente se considera pensable. Esto impide acceder al pensamiento sistémico, holístico e integrado, y trascender el pensamiento binario que opone teoría a práctica, local a global, conocimiento científico a conocimiento o saber común. Por esto, el planteamiento de Morín invita a pensar integradamente, y a considerar que los problemas no son meramente locales, y que si así lo fueran sería necesario conectarlos con la vida global, especialmente hoy cuando la relación entre lo global y lo local es tan interdependiente que nos pensamos como globales en nuestra propia geografía local.

Desde la perspectiva de Nicolescu (1996, p. 37) se postula que es importante trascender los marcos disciplinarios y pensar más allá de las disciplinas. Se trata, según su perspectiva, de comprender el mundo integradamente disolviendo las limitaciones y delimitaciones del pensamiento disciplinario. Por lo tanto, para este autor es fundamental superar la lógica binaria que opone una disciplina científica a una no científica. Desde su punto de vista, la lógica de producción del conocimiento hoy ha superado la compartimentación disciplinaria creando nuevas formas de organización y de relación entre ellas. En este sentido, la integración, la inclusión y el diálogo son la punta de lanza de la cultura transdisciplinaria (Nicolescu, 1996). Este planteamiento se orienta hacia lo que este autor llama la "unidad del conocimiento". 
No es claro si este propósito de unificar el conocimiento sea viable, pues sabemos que desde muy temprano este se dividió en campos que conocemos como disciplinas, y las disciplinas son la expresión de la división del trabajo del conocimiento. Más que lograr la unidad del conocimiento se trata de superar la fragmentación y de pensar, por ejemplo, que debiera haber un diálogo entre el conocimiento científico y el conocimiento no científico, o entre los métodos de las diversas ciencias. Es evidente que hoy se dan nuevas formas de producción de conocimiento que no tienen una base estrictamente disciplinaria, aunque reconocen la existencia de las disciplinas. Por esto, como dice Nicolescu (1996), la integración, la inclusión y el diálogo son el "trigger" de la cultura transdisciplinaria. Esta difiere teórica y metodológicamente de la cultura disciplinaria. En esta se privilegia la división entre disciplinas. Cada una tiene su propio territorio epistémico, el cual entra en el juego de las jerarquías verticales socialmente producidas. Por su parte, en la cultura transdisciplinaria se debilitan los límites entre las disciplinas. Este debilitamiento da origen a nuevos campos de conocimiento, a nuevos estudios que no reconocen una identidad disciplinaria. En dicho caso se promueve el diálogo no disciplinario, se busca la conexión de los conceptos, y el encuentro transdiscursivo que recrea la hibridez de la narratividad (etnográfica, biográfica o autobiográfica) con la teoría ${ }^{1}$. Esto se asimila a la perspectiva de Clifford Geertz (1980) con su famoso concepto "blurred genres" o la refiguración del pensamiento social.

Como puede observarse, es posible transitar de una cultura disciplinaria a una cultura transdisciplinaria que posibilite otras formas de organización del conocimiento y otras formas de investigación. Es importante aclarar que el tránsito a la transdisciplinariedad no significa lograr la unidad del conocimiento. Este es un asunto muy difícil que escapa a su naturaleza. Es importante preguntarse cómo podría unificarse el conocimiento científico con el conocimiento común, cuando históricamente estas dos formas de conocimiento poseen estructuras diferentes. Quizás el avance más importante en materia de transdisciplinariedad se ha producido alrededor del debilitamiento de los límites entre las disciplinas que ha conducido a hibridajes entre estas. Así, por ejemplo, de la misma manera que un reservorio recibe aguas de diferentes afluentes que en

1 Un ejemplo de esta hibridez esta dada en la obra de Richard Sennett. 
él se hibridan, podemos pensar el espacio transdisciplinario figurativa o metafóricamente como un reservorio discursivo en el cual convergen discursos de diferentes disciplinas, que se hibridan y producen nuevos conocimientos, que no pertenecen a ninguna disciplina. Esto puede extenderse a las relaciones entre conocimiento común y conocimiento científico al producirse un espacio que podría denominarse diálogo de saberes que daría origen a nuevas narrativas, como ocurre en la actualidad en las ciencias sociales (Sennett, 2000). A partir del ejemplo mencionado, podemos pensar la transdisciplinariedad en términos del hibridaje de discursos que no reconocen una identidad disciplinaria. Como ejemplos se pueden mencionar las ciencias ambientales, el género, la comunicación e, inclusive, el discurso de la educación.

A partir del planteamiento recuperado anteriormente de Nicolescu (1996), es posible comprender de otra manera el discurso del género, cuyo tratamiento ya no se basa en la clásica oposición binaria masculino/ femenino, dado que como hecho social tiene realizaciones plurales, y como objeto de estudio ha devenido transdisciplinario. Desde esta perspectiva, se puede asumir el campo de estudios del género como un campo compuesto por una red discursiva que conecta conceptos de diferentes disciplinas (lingüística, sociología, semiótica, derecho, psicoanálisis) sin jerarquía entre ellas y donde el nuevo discurso no tiene una identidad disciplinaria. Esto, como se dijo atrás, significa la transdisciplinariedad: ir más allá de la disciplina, más allá del pensamiento disciplinario, indagar en los límites epistémicos, articular campos de conocimiento y construir nuevos espacios discursivos más allá de las disciplinas singulares.

El concepto de transdisciplinariedad es clave en la construcción de nuestra argumentación en favor de la transversalidad curricular propia de un modelo de formación transversal. La razón básica es que el conocimiento en el campo educativo se ha movido y transformado a imagen y semejanza de la dinámica del campo de producción del conocimiento donde, como se ha dicho, ha predominado la perspectiva disciplinaria. De allí que, por ejemplo, la asignatura sea una especie de imagen microscópica de las disciplinas. Sin embargo, el desarrollo del conocimiento ha conllevado nuevas formas de relación entre las disciplinas que han favorecido su enriquecimiento sin que se debiliten los límites entre ellas. Es el caso de la interdisciplinariedad, donde puede darse la cooperación en términos de convergencia metodológica como 
ocurre en cierta cooperación investigativa. Es claro que las nuevas formas de producción de conocimiento han dado origen a nuevos discursos que no reclaman identidad disciplinaria y demandan la existencia del conocimiento sin las fronteras estables de las disciplinas.

Comprender estas transformaciones es una necesidad teórica y metodológica para entender el cambio curricular que va de las asignaturas aisladas, que son una especie de metáfora de la disciplinariedad, a formas nuevas de organización del conocimiento educativo basadas en los principios de integración y transversalidad, que conocemos como currículo integrado, donde se debilitan fuertemente los límites entre los contenidos para dar paso a nuevas formas de su organización. El concepto de currículo integrado no se refiera a la reunión o a la pluralidad de contenidos que no se relacionan entre sí. El principio básico que regula la existencia de un currículo integrado es el debilitamiento de los límites. A este currículo, distorsionadamente se le denomina, inclusive por académicos universitarios, currículo interdisciplinario.

Como puede observarse, disciplina y currículo pertenecen a dos campos diferentes: las disciplinas se ubican en el campo de producción de conocimiento, el currículo se ubica en el campo de reproducción de conocimiento, y es claro que los límites entre estos dos campos se han mantenido rígido inclusive en el siglo XXI, a pesar de las posturas de nuevos teóricos sobre el cambio en la naturaleza del conocimiento. De allí que para pensar en la transversalidad y en la transversalidad curricular se requiera primero partir del análisis de la transdisciplinariedad, lo cual no significa que estos conceptos pertenezcan al mismo plano de análisis.

\section{Sobre la transversalidad}

Examinemosahoraelconcepto detransversalidad.Transdisciplinariedad y transversalidad parecen ser equivalentes. Sin embargo, se refieren a cuestiones, objetos y problemas diferentes. La transversalidad expresa la manera en que conceptos, problemas, situaciones viajan de un campo a otro, o entre diversos campos. Esto hace que no permanezcan anclados en un solo espacio disciplinario, o a un campo o contexto específico. El tránsito de conceptos o problemas de un campo a otro expresa la posibilidad que estos vayan más allá de su espacio original. Por esta razón, podemos decir, por ejemplo, que el género como concepto o como problema es transversal 
a múltiples disciplinas, está por encima de ellas, no pertenece a ninguna de ellas. Cuando se dice que la transversalidad no es la relación amistosa entre campos, lo que se quiere significar es que no basta el diálogo entre las disciplinas para que un estudio sea transdisciplinario. Todos sabemos que es muy común que las disciplinas dialoguen sobre un problema. Sin embargo, ese diálogo puede ser multidisciplinario, lo cual quiere decir que cada disciplina dialoga desde su territorio sin ceder o debilitar su identidad. Cada disciplina dice algo del problema, y puede que haya convergencia, pero habla desde su punto de vista, de manera independiente.

La transversalidad permite el diálogo y la construcción de nuevos discursos y prácticas sobre nuevos problemas. Esto se debe a que los problemas no pertenecen a ninguna disciplina, porque están más allá de las disciplinas, así estas aporten nuevas comprensiones y, de esta manera, den origen a nuevos conceptos o discursos, como es el caso de los estudios sobre el género, el medio ambiente, o la desnutrición. Una importante posición alrededor de la transversalidad es la de Diego Bermejo (2005), quien en su libro Postmodernidad, pluralidad y transversalidad plantea este concepto en términos de tránsito, entrecruzamiento o articulación de paradigmas que flexibilizan sus límites. Desde su punto de vista,

El concepto de transversalidad, asociado al de pluralidad, permite comprender que los paradigmas no son unidades aisladas, sino que su constitución es a su vez transectorial o intersectorial, hecha de préstamos, contactos, cruzamientos, desplazamientos entre paradigmas de diferentes tipos de racionalidad. Su estructura es conectiva y multitransversal, interparadigmática (p. 20).

En este sentido, se entiende por transversal "el modo de operar por tránsitos pluridireccionales y plurimodales entre complejos diferentes" (p. 61). Se puede argumentar, entonces, que la transversalidad presupone tránsito de conceptos o discursos de un lugar a otro, entrecruzamiento de discursos o articulación de ellos. Para que esto ocurra resulta oportuno que se flexibilicen los límites entre los conceptos, los campos, los discursos, provengan o no de las disciplinas. El planteamiento de Bermejo (2005) resulta muy interesante porque asocia pluralidad con transversalidad. En este sentido, las disciplinas, si bien son unidades aisladas, su desarrollo latente es, a su vez, inter y transectorial, hecho de préstamos, contactos y cruzamientos con otras disciplinas. Con base en este planteamiento, se puede asumir que la estructura de las disciplinas es conectiva y multitransversal. Esto significa que los conceptos de un campo pueden conectarse con aquellos de otros campos, 
e inclusive, viajar más allá de ellos. En términos curriculares y pedagógicos, esto significa que cuando trabajamos sobre un problema concreto debemos asumir una visión de conjunto, integrada o sistémica.

Desde otra perspectiva, la posición de Deleuze y Guattari (1993) aporta elementos importantes para la comprensión de la transversalidad. Conceptos como conexión, multiplicidad, líneas, mapas, tienen una relación directa con su significado de transversalidad, y pueden ser utilizados para entender mejor la transversalidad curricular. En su libro ¿Qué es la filosofía? Deleuze y Guattari (1993) plantean que no hay concepto propio, que los conceptos se construyen en red o conexión con otros conceptos. En este sentido, podríamos decir que un concepto presupone relaciones transversales con otros. Los estudios de Deleuze y Guattari están orientados a producir un nuevo tipo de organización transversal basado en el denominado "paradigma rizomático". Este paradigma remite a la multiplicidad de una estructura. El rizoma es una metáfora para explicar la discontinuidad y ausencia de jerarquía. En su obra denominada Mil Mesetas, Capitalismo y Esquizofrenia (1988), estos autores hacen una exposición sobre lo que entienden por rizoma, que es relativamente compleja. Allí apropian el significado biológico de rizoma, el cual se refiere a un tallo subterráneo que tiene formas muy diversas, con una extensión ramificada en todos los sentidos. Este significado se apropia metafóricamente para mostrar la importancia de la articulación, conexión, e integración de conceptos y para demostrar que un concepto es plural porque está hecho de muchos conceptos.

Dada su importancia, el concepto de transversalidad también ha sido apropiado por agencias externas a la educación para introducirlo en este campo. Desafortunadamente, como muchos conceptos que se recontextualizan en el campo educativo terminan siendo distorsionados, segmentados y marginales. Como ejemplo se pueden colocar los ejes transversales ligados a la educación en valores (convivencia, interculturalidad, paz, salud, sexualidad, etc.), que se si bien se consideran temas relevantesparala formación integral, están presentesmarginalmente en el currículo, y se resuelven a través de asignaturas adicionales que poco interés tienen para los estudiantes. Esta visión distorsionada de la transversalidad obliga a pensarla en términos estructurales y sistémicos, pues no solo de trata de configurar un currículo flexible e integrado sino, también, formar sujetos flexibles, transversale y competentes Al respecto, Bermejo (2005) plantea que "en la idea del sujeto transversal las diversas competencias del sujeto están internamente conectadas y entretejidas" ( $p$. 
121). Esto le permite al sujeto participar de manera abierta y flexible en la multiplicidad de formas de vida propias de la pluralidad sociocultural actual.

\section{La transversalidad curricular}

Los planteamientos anteriores sirven de base para la comprensión y análisis de la transversalidad curricular. Existen muchos problemas en la sociedad que son multidimensionales y que pueden ser estudiados inter o transdisciplinariamente. Cuando esos problemas llegan al currículo existe la tendencia a incorporarlos de manera aislada a las asignaturas, sin considerar que no pertenecen a ninguna de ellas y que pueden ser estudiados por todas las asignaturas del currículo. Cuando los problemas seleccionados no pertenecen a ninguna asignatura y pueden ser estudiados por todas, hablamos de problemas transversales, donde ya no se dialoga desde las asignaturas aisladas sino desde el conocimiento o el saber. Por esta razón, cuando hablamos de currículo transversal o de transversalidad curricular nos referimos a un currículo que está organizado a partir de la flexibilización de los límites entre los contenidos, lo cual permite que la organización de estos se despliegue bajo la forma de temas o problemas transversales, cuyo estudio es integrado o, metafóricamente hablando, transdisciplinario.

Desde este punto de vista se puede definir un currículo transversal como aquel en el cual los contenidos de aprendizaje no pertenecen a ningún área o asignatura, porque viajan a través de éstas. Por esta razón, pertenecen a todas las áreas de conocimiento y no se ubican en ninguna de ellas. Generalmente se refieren a temas, problemas, situaciones, casos, que pueden tener una mirada integrada o sistémica de su conocimiento ${ }^{2}$. Pensemos en la violencia como ejemplo de transversalidad curricular. En

2 Metodológicamente se debe distinguir entre integración y transdisciplinariedad. Generalmente, estos términos se usan indiscriminadamente. Sin embargo, transdisciplinariedad pertenece al campo de las disciplinas e integración pertenece al campo del currículo. En este sentido, no existe un currículo interdisciplinario porque no pertenece al campo de las disciplinas. Lo que existe es un currículo integrado que apela al conocimiento para la comprensión de todos sus objetos. Es importante diferenciar teórica y metodológicamente dos campos: el campo de las disciplinas y el campo del currículo. Esta diferencia crea una semántica propia para cada campo. Ahora bien, el hecho de que en el sentido común el término integración pueda extenderse a cualquier contexto, no significa que esto pueda desplazarse al lenguaje teórico donde los conceptos no deben presentar ambigüedad. 
la violencia hay conexión, transitividad y articulación con respecto a la familia, al género, la niñez o la comunidad. Esto puede producir una serie de relaciones entre problemas que se viven en diversos grupos sociales, los cuales pueden recibir un tratamiento transdisciplinario. Otro ejemplo de transversalidad curricular es el que se refiere al medio ambiente. El medio ambiente como problema actual es multidimensional. Su estudio no pertenece a una sola disciplina porque está presente en la mayoría de ellas. El medio ambiente es un problema político-económico-socialcultural-científico-tecnológico, pero también natural. Hoy su deterioro debe ser objeto de compromisos integrados. El medio ambiente ha dejado de ser tratado como un objeto natural para convertirse en una construcción social y como tal sus problemas se han vuelto transversales. De allí que sea objeto de compromisos políticos (políticas medioambientales); que reciba el impacto del desarrollo de la producción económica y sociocultural, porque está afectado por el desarrollo de la sociedad y por los patrones de consumo moderno cuyo excedente es la basura que alcanza volúmenes insospechados en el planeta. En consecuencia, su estudio es transdisciplinario.

Con base en este ejemplo se puede establecer una diferencia entre transdisciplinariedad y transversalidad (Nicolescu, 1996). Por una parte, los conceptos sobre el medio ambiente pueden ser transdisciplinarios; por la otra, sus problemas pertinentes pueden considerarse transversales. Esto nos permite plantear la existencia de dos planos diferentes de análisis: el plano de los problemas transversales, y el del conocimiento científico transdisciplinario. Estos pueden describirse como dos planos analíticos: el plano del conocimiento (de las disciplinas) y el plano de los problemas sociales. En el plano del conocimiento encontramos conceptos transdisciplinarios con los que se puede analizar el discurso del medio ambiente, del género, de la democracia, de la violencia, y que se fundamentan en disciplinas que convergen en su construcción. En el plano de los problemas encontramos que estos son transversales, transitivos. Esto quiere decir, en otros términos, que el discurso sobre un problema puede configurar un discurso transdisciplinario, y que el problema que se estudia presenta transversalidad con otros problemas.

Otro ejemplo puede ser el de la comunicación. Si se piensa en un programa de formación profesional de comunicación, el currículo debiera ser un currículo transversal, integrado, y no un currículo lleno de materias 
agregadas. La transversalidad curricular aquí implicaría seleccionar una serie de problemas transversales de la comunicación e investigarlos en grupos o equipos, esto es, colegiadamente. Esto permitiría, por una parte, transformar la organización curricular de asignaturas aisladas en problemas transversales $\mathrm{y}$, por la otra, mostrar las formas diversas bajo las cuales se presentan los conceptos descriptivos y analíticos sobre la comunicación. El efecto de la organización curricular permitiría, en este caso, la construcción de nuevas formas de aprendizaje colectivo, socializado, donde la unidad organizativa de los estudiantes no sea el estudiante aislado, sino un equipo de investigación.

Como puede observarse, la transversalidad curricular afecta no solo la selección de los contenidos curriculares sino también su organización. Este principio puede extenderse a los estudiantes. En este caso se trata de crear nuevas formas de organización estudiantil que posibiliten mayor interacción entre los aprendices y que, al mismo tiempo, faciliten el desarrollo del aprendizaje socializado, participativo y colaborativo (Diaz Villa, 2007). Son diversos los ejemplos que pueden presentarse sobre transdisciplinariedad y transversalidad. En uno u otro caso se trata de ir más allá de los límites creados entre las disciplinas, por una parte, y más allá del tratamiento fragmentado y por demás especializado de los problemas. La transversalidad presupone flexibilidad, integración, hibridez, inexistencia de jerarquías. Estos mismos rasgos pueden considerarse en la transdisciplinariedad en otros escenarios como el de la salud, la contaminación, el calentamiento global, la comunicación, el medio ambiente, etc. Si consideramos por ejemplo la salud, no podemos pensarla de manera unilateral, como ocurre regularmente en la medicina curativa especializada, porque la salud está asociada a factores o problemas sistémicos que son causa de su bienestar o su deterioro y, en consecuencia, de su calidad. Aquí podemos ver dos aspectos de la salud. El aspecto científico de su estudio que debiera ser transdisciplinario, y no simplemente multidisciplinario, y el aspecto relacionado con los problemas que en ella se generan que son transversales. Cada problema de salud está conectado con otro u otros problemas y así sucesivamente, de una manera sistémica y holística.

En síntesis, la diferencia entre transdisciplinariedad y transversalidad es muy sutil. Si bien la transdisciplinariedad pertenece al campo de las disciplinas que debilitan sus límites, se integran o se hibridan para formar nuevos campos de conocimiento que no son disciplinarios, el proceso del 
tránsito de los conceptos entre diferentes disciplinas es transversal. Ahora bien, una vez que un concepto se produce con la participación de diferentes disciplinas que convergen en sus aportes teóricos o metodológicos se considera transdisciplinario. Por su parte, la transversalidad pertenece al orden de los problemas o al plano de los problemas pues, como se dijo atrás, su análisis y solución cruza diferentes disciplinas.

Ahora bien, cuando nos referimos al currículo es conveniente hablar de transversalidad curricular o de currículo transversal. Metodológicamente, es posible distinguir entre el campo de las disciplinas y el campo del currículo. El segundo es subsidiario del primero, en la medida en que ambos campos poseen estructuras diferentes.

Si apelamos a la metáfora de Deleuze y Guattari (1988), el currículo transversal también puede entenderse y definirse como un currículo rizomático ¿Por qué se denomina rizomático? Porque no responde a jerarquías entre los conocimientos seleccionados, esto es, no responde a la clásica organización vertical del conocimiento educativo expresada en tiempos asignados a las materias, en jerarquías de estas socialmente construidas (obligatorio-electivo) y a la lógica de los discursos disciplinarios que se conocen cotidianamente como las asignaturas.

En la semántica de Deleuze y Guattari (1993) se puede decir que un currículo rizomático es aquel cuyos contenidos presentan articulaciones, provienen de múltiples fuentes, entran en relaciones horizontales, no están territorializados, no reproducen jerarquías, tienen formas diversas de organización y reorganización, y se fundamentan en la transitividad -un contenido o conocimiento remite a otro, y a otro, y así sucesivamente en una cadena abierta-. Un currículo rizomático responde, por lo tanto, al principio de conexión y heterogeneidad, se basa en una selección no condicionada por la fragmentación disciplinaria, puede organizarse de múltiples formas y responde a las demandas de diversos contextos. Sobre la base del pensamiento rizomático de Deleuze y Guattari (1993), podemos considerar que la unidad básica del currículo rizomático es el "rizoma curricular", esto es, una unidad de contenido abierta a múltiples articulaciones, conexiones, contextos y posibilidades interpretativas.

En el ámbito internacional, la UNESCO ha planteado la necesidad de incorporar los ejes transversales en los currículos. Entre algunos de los 
que considera pertinentes están: la juventud, el agua, el cambio climático, la libertad, los derechos humanos, la responsabilidad social, ética y la solidaridad (UNESCO, 1998). Todos estos problemas presuponen un pensamiento transversal que implica flexibilidad, integración, hibridez, pluralidad, conectividad y horizontalidad $y$, en última instancia, el debilitamiento de los límites. Sin embargo, sería conveniente trascender el carácter marginal que tienen, ya que están limitados a la formación en valores, y no abarcan la totalidad del currículo.

Por esto, el tema del debilitamiento de los límites es importante para comprender la flexibilidad, la integración y, en especial, la transversalidad. Cuando los límites son flexibles hay transitividad, capacidad de circulación de un espacio a otro, de un lugar a otro, de un concepto a otro, o de una disciplina a otra. Observamos, entonces, que el poder transitar a través de los límites flexibles de las disciplinas, traspasarlos, puede entenderse en términos de transversalidad. Así mismo, la conexión, articulación, entre problemas puede entenderse mejor en términos de transversalidad.

En síntesis, la transversalidad se ha vuelto un tema recurrente en la educación. Desde la educación preescolar hasta la universidad se plantea la necesidad de crear currículos transversales, integrados, o rizomáticos ${ }^{3}$. Sin embargo, hay cierta distancia entre proponer currículos transversales y expresar de otra manera la transversalidad curricular que vaya más allá de las definiciones comunes, presentes en muchos textos escolares. Este es un problema que afecta a docentes y estudiantes, pues la pedagogización de la transversalidad minimiza su sentido macrosocial, y la importancia de abordar el pensamiento de los problemas en el eje macro-micro. ¿Qué significa esto? Desde la perspectiva transversal significa que los problemas por microscópicos que sean obedecen a macro determinaciones o macro estructuras. Un ejemplo que puede ilustrar esto es el problema de las basuras. Una teoría de la basura tendría que plantear su carácter global, las causa globales de su existencia, la economía desbordada de

3 Desde la perspectiva que aquí se plantea estos términos son equivalentes.

4 Los conceptos transversal, rizomático, integrado presentan una equivalencia semántica debida a la hibridez conceptual que han producido los discursos postmodernos que no han sido exentos del fenómeno de la pluralización conceptual. Hoy todo está anudado, conectado, interrelacionado. Somos nodos en las redes sociales, y asumimos una multiplicidad de roles. Bermejo (2005) coloca como ejemplo el politeísmo de valores. 
producción de bienes y servicios y el consumo masivo que ha conducido a que los excedentes sean mayúsculos y conduzcan a la contaminación de suelos, aguas, aire, y al deterioro de la salud. Por esta razón, la basura no es un problema local, de micro contextos, como tampoco es un problema producido individualmente. Los contextos macroscópicos de la economía global, el consumo global, ha hecho que la basura se convierta en un macro problema. Es, en esencia, un problema transversal ${ }^{4}$.

\section{Transversalidad y modelos de formación}

Cualquiera que sea el enfoque del currículo (transversal, rizomático, integrado, contextualizado), su pedagogización ha conducido a procedimientos que no van más allá de la simple correlación de conceptos, problemas, o temas. Es evidente que este tipo de "integración" de cierto nivel no cambia el pensamiento de los estudiantes. De allí la importancia de construir modelos de formación alternativos que efectivamente permitan aproximar nuevas realidades, nuevas prácticas $y$, sobre todo, nuevas formas de pensar y comprender las realidades que vivimos en la actualidad. Aquí juega un papel muy importante el concepto de modelo y el de modelo de formación.

El concepto de modelo es relativamente complejo. El término posee diferentes significados que dependen del contexto donde es usado. Si bien su definición más corriente es la que hace referencia a la representación en pequeño de alguna cosa, esa representación varía de conformidad con el diseño que se elabora por quienes lo construyen (Badiou, 1968). Así, por ejemplo, en la Arquitectura tenemos el modelo en miniatura de un edificio, o de conjunto residencial. El modelo del edificio lo representa, pero no es el edificio real. En la escultura un modelo es alguien que se debe copiar. Un modelo económico representa un proceso a seguir que sirve de control sobre las actividades económicas. Así tenemos modelos en las Matemáticas, la Física, la Química, etc.

Por esto Badiou (1968) plantea que "un modelo posee una existencia teórica y formal. Se organiza sobre estructuras y simula una realidad y la describe y explica a través de conceptos" (pp.21-49). De esta manera, es posible encontrar modelos en la generalidad de la producción teórica. En ellos hay convergencia alrededor de la idea de que un modelo es un sistema conceptual abstracto, o una representación abstracta de la 
experiencia. Desde la perspectiva de este autor, un modelo posee una existencia teórica y formal. Simula una realidad y la describe y explica a través de conceptos. Esto significa que un modelo no es la realidad misma. Solo la simula, la proyecta, la representa. Por esta razón, como se planteó atrás, es posible decir que un modelo corresponde al dominio de los conceptos y en este sentido es abstracto.

Ahora bien, cuando se hace referencia al campo educativo se observa que hay una especie de desgaste del concepto debido a que se ha extendido a diversos subcampos. Esto ha producido una confusión semántica en relación con los modelos que adoptan las instituciones. Así es posible encontrar una diversidad de modelos que han tenido un efecto en el debilitamiento del concepto y que han creado confusión entre los docentes y administrativos. Todos ellos tienen alguna similitud pues se refieren a la educación, pero se diferencian en el objeto que representan o simulan. De allí la necesidad de establecer articulaciones entre estos modelos, así como definir su autonomía. La siguiente figura intenta representar las relaciones de los modelos que hasta hoy circulan en el campo educativo.

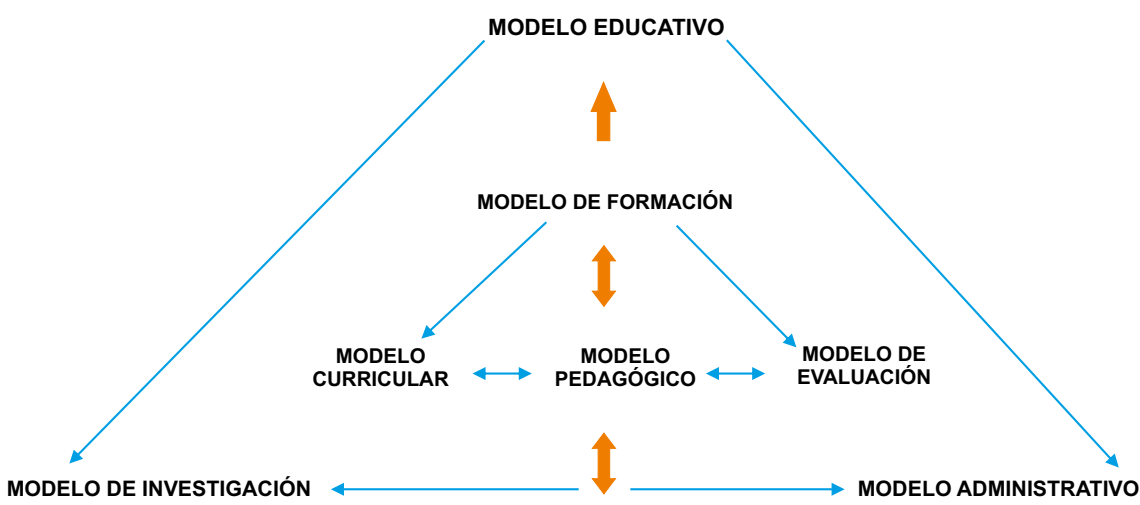

Figura 1. Relaciones de los modelos en el campo de la educación.

Fuente: Elaboración propia.

Como se puede apreciar en la figura, hay niveles de relaciones de articulación entre estos modelos. Sin embargo, todos confluyen en el modelo educativo porque en él se resumen todas las políticas, lineamientos, estrategias y posibles prácticas de orden institucional. A su vez, el modelo educativo se expresa en tres modelos interrelacionados 
-el modelo de formación que subsume el modelo de investigación (que simula los procesos investigativos) y el modelo administrativo. Sin bien hay una relación sistémica entre estos, la mayoría de las instituciones educativas no consideran sus articulaciones y solo incorporan en toda la vida institucional un modelo, que generalmente corresponde al pedagógico.

El modelo de formación resulta de particular interés en el presente análisis. Simula todo el proceso formativo y tiene como propósito mostrar cómo este opera en una institución. Un modelo de formación parte del concepto que históricamente, de manera simplificada, significa a grandes rasgos guiaru orientar al aprendiz en su desarrollo y crecimiento académico, personal, social y político. Se trata de la representación abstracta de una experiencia educativa que se basa en conceptos y relaciones que guían la acción. Toda experiencia formativa implica la articulación de diversos componentes: actores, unidades académicas, discursos (currículo) y prácticas (pedagógicas, evaluativas, administrativas). La siguiente figura ilustra los componentes de las categorías constitutivas de un modelo de formación.

MODELO DE FORMACIÓN ARTICULA

INSTITUCIONES

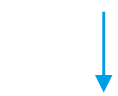

La escuela en sus diferentes niveles
SUJETOS

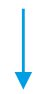

Docentes

Administrativos

Estudiantes

Padres
DISCURSOS

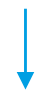

Conocimientos
Enfoques
Teorías

Métodos
PRÁCTICAS

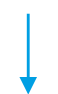

Curriculares

Pedagógicas

Evaluativas

Administrativas

Figura 2. Componentes de las categorías constitutivas de un modelo de formación.

Fuente: Elaboración propia.

Como puede observarse, el modelo articula un conjunto de componentes que pueden clasificarse en internos y externos que son interdependientes debido a su profunda articulación. Los componentes internos se refieren a la naturaleza propia de modelo, esto es, a la 
organización interna de los elementos que lo constituyen y que están interrelacionados. Los componentes internos del modelo son el currículo, la pedagogía y la evaluación, y se corresponden con los elementos que orientan directamente las prácticas pedagógicas de formación. Estos elementos son constantes y pueden permanecer por mucho tiempo sin tener variaciones o cambios. Así, por ejemplo, el currículo de un programa puede permanecer sin modificarse hasta una década y depende de principios institucionales que son externos. La pedagogía está definida en ocasiones institucionalmente a partir de ciertos enfoques o teorías que dominan en el escenario educativo, a pesar del sello personal que le imprimen los docentes; y la evaluación está regida por normas consignadas en reglamentos específicos. Podemos agregar que esos elementos internos son constantes. Por su parte, los componentes externos del modelo se refieren a los contextos o entornos social, cultural, económico, y político. Ellos tienen relaciones de interacción con los elementos internos. Como ejemplos se proponen las políticas educativas globales y nacionales que orientan el quehacer de las instituciones; los discursos diversos (sobre el aprendizaje, la enseñanza, las competencias, el niño, el maestro, etc.); la transversalidad (ejes transversales propuestos por la UNESCO); el discurso de la flexibilidad (flexibilidad curricular, flexibilidad pedagógica, flexibilidad académica, flexibilidad administrativa) ${ }^{5}$.

En síntesis, se puede afirmar que el concepto de modelo de formación implica conocimientos, instituciones, sujetos, sistemas, normas pedagógicas, condiciones legales, jerarquías que fijan límites al quehacer educativo. Es la dinámica de estos componentes la que directa o indirectamente regula el proceso de formación. Por eso se ha planteado a lo largo de este artículo que un modelo de formación simula el proceso formativo.

El concepto modelo de formación puede considerarse la base para la construcción del concepto modelo de formación transversal. Este tiene como propósito producir modalidades de formación alternativa basadas en la transversalidad. Se ha dicho que estos dos principios son fundamentales para la transformación del currículo y la pedagogía y, en

5 Es importante tener en cuenta que estos discursos no se crearon en la educación. sino que pertenecen a otros campos. Su tránsito a la educación se conoce, en términos de Bernstein (1998) como recontextualización. Véase Díaz Villa (2007). 
consecuencia, en la transformación de las modalidades de aprendizaje centradas en el individuo y no en el grupo. Por esto es importante que un modelo de formación transversal, independientemente de sus realizaciones contextuales, se base en los siguientes postulados:

- Integración de los principios, contextos y prácticas de investigación entre los campos de conocimiento.

- Integración en el currículo de problemas concretos susceptibles de estudio transversal.

- Énfasis en la investigación, la comprensión, e interpretación de los problemas y su posible solución de manera socializada.

- Generación de un aprendizaje integrado que favorezca el diálogo de conocimientos y saberes de los diferentes campos epistémicos, y los articule a soluciones alternativas de problemas del entorno.

- Desarrollo creciente de las competencias que subyacen a la investigación (epistémica, discursiva, y textual).

- Énfasis en el aprendizaje socializado, participativo y colaborativo (Díaz, 2007).

En consecuencia, estas características se extienden igualmente al currículo, y a la integración de problemas concretos susceptibles de estudio transversal. Esto favorece la investigación, la comprensión e interpretación de los problemas y su posible solución de manera socializada. En cierto sentido, el currículo intrínseco a un modelo de formación transversal recupera los principios de un currículo rizomático, el cual no resulta de la suma de problemas aislados, sino de las conexiones o interrelaciones que se dan entre ellos, y de la manera como se resuelven a través de la integración de conocimientos. En la misma forma que el rizoma está relacionado con un mapa que es producido, construido, siempre desmontable, conectable, alterable, modificable, con múltiples entradas y salidas, el currículo rizomático conecta, altera o modifica los contenidos. Los conceptos de conectable, alterable, modificable, con múltiples entradas y salidas, son propios de un currículo que no está impulsado por las entradas predefinidas de los expertos, sino que se construye y se negocian en tiempo real con los aportes de quienes participan en el proceso de aprendizaje (Santamaría, 2012). Es dentro de ese conjunto de transformaciones que puede entenderse la transversalidad y la transversalidad de un modelo de formación y, a partir de esto, la producción de otras perspectivas investigativas, fundamentadas en la generación de 
nuevos contextos de interacción, que promuevan el acceso comprensivo al conocimiento y a la solución de problemas de manera socializada y ampliamente interactiva.

\section{Conclusiones}

Este documento ha desarrollado algunos aspectos conceptuales relacionados con la transdisciplinariedad, la transversalidad y el currículo. Se partió del análisis de las relaciones entre las disciplinas y se consideró la transdisciplinariedad como el eje de la discusión alrededor de los procesos de producción del conocimiento. También se consideró el debilitamiento de los límites como la base de la transdisciplinariedad y la transversalidad. Así mismo se estableció su influencia en la formulación del currículo transversal, integrado o rizomático en el cual se desvanece la idea de jerarquía entre los contenidos y surge un enhebrado de relaciones asociadas a una visión integradora del conocimiento.

En contraposición a una concepción del conocimiento rígidamente delimitado, se consideró la potencia de la transversalidad como una expresión de la integración de diferentes niveles de realidad presentes en el currículo y, a través de estos, a los procesos de formación. Esto condujo a formular descriptivamente una estructura articulada de la formación mediada por la interrelación (o interdependencia) de modelos intrínsecos al campo educativo que operan en diferentes niveles y que confluyen alrededor de lo que se concibe como modelo de formación.

En síntesis, se concluye que la transversalidad juega un papel fundamental en la medida que no obedece a la racionalidad inherente a las posiciones clásicas disciplinarias que alimentan las oposiciones, los territorialismos y la reproducción de límites rígidos entre los campos epistémicos. En oposición a esta perspectiva, la transversalidad es inherente a la integración y se opone radicalmente a la jerarquización en las formas de organización. A la transversalidad subyace la cultura de la integración. La trascendencia de la transversalidad en el campo educativo favorece la producción de modalidades curriculares cuyas bases son la conexión, la articulación, el hibridaje de los contenidos, todo esto bajo el principio básico de la integración que surge del debilitamiento de los límites. 
Este documento puede verse como una exhortación al fortalecimiento de modalidades alternativas de pensar el currículo que vayan más allá de las formas organizativas actuales, las cuales, en materia formativa, riñen con la cultura de una sociedad caracterizada por el cambio de un orden jerárquico, a una cultura en la cual el sincretismo es un principio que domina todos los escenarios de organización e interacción. De la misma manera que el efecto de este sincretismo ha sido la remodelación del yo (Bell, 1996), las fronteras rígidas intrínsecas al currículo pueden dar paso a nuevas formas de exploración sobre los arreglos del conocimiento educativo y, a través de estas, a nuevas formas de exploración, pensamiento y producción de conocimiento.

\section{Referencias Bibliográficas}

Badiou, A. (1972). El concepto de Modelo. Bases para una Epistemología Materialista de las Matemáticas. Buenos Aires: Siglo XXI Editores.

Becher, T. (2001). Tribus y Territorios Académicos. La Indagación Intelectual y las Culturas de las Disciplinas. Barcelona: Editorial Gedisa.

Bell, D. (1996). Las contradicciones culturales del capitalismo. Madrid: Alianza Editorial.

Bermejo, D. (2005). Posmodernidad, pluralidad y transversalidad. Barcelona: Anthropos.

Bernstein, B. (1977). Clasificación y enmarcación del conocimiento Educativo. En Class, codes, and control, Volumen 3. Towards a theory of educational transmitión. London: RKP, pp. 202-230.

Deleuze, G. y Guattari, F. (1988). Mil mesetas. Capitalismo y Esquizofrenia. París: Editorial Pre- textos.

Deleuze, G. y Guattari, F. (1993). ¿Qué es la filosofía?. Barcelona: Anagrama.

Diaz, Villa, M. (2007). Lectura Crítica de la Flexibilidad. Vol I. La Educación Superior frente a los retos de la flexibilidad. Bogotá: Cooperativa Editorial Magisterio.

Dogan, M. (2003). Las nuevas ciencias sociales: grietas en las murallas de las disciplinas. Recuperado de: https://metodologiasdelainvestigacion. wordpress.com/2010/11/20/las-nuevas-ciencias-sociales-grietas-en-lasmurallas-de-las-disciplinas-mattei-dogan-unesco/

Geertz, C. (1980). Blurred Genres: the refiguration of social thought. The American Scholar, Vol. $49 \mathrm{~N}^{\circ} 2$ (1980), pp. 165-179

Moreno Bayardo, M. G. (2005). Potenciar la educación. un currículum transversal de formación para la investigación REICE. Revista Iberoamericana sobre Calidad, Eficacia y Cambio en Educación, Vol. 3, № 1, pp. 520-540. Morín, E. (1990). Introducción al pensamiento Complejo. Madrid: Gedisa. 
Nicolescu, B. (1996). La Transdisciplinariedad Manifiesto. París: Ediciones Du Rocher.

Saur, D. (2013). Entrevista a Rosa Nidia Buenfil Burgos. Eclecticismo y transversalidad en la investigación educativa. En Propuesta Educativa 39, 5-12.

Sennett, R. (2000). La corrosión del carácter. Barcelona: Anagrama.

Santamaría, F. (15 de febrero 2012). Reflexiones sobre ecologías y espacios del aprendizaje, análisis del aprendizaje y análisis de las redes sociales. visualización de datos. Big Data y otros temas emergentes. Blog de Fernando Santamaría. educación rizomática $\mid$ Blog de Fernando Santamaría (fernandosantamaria.com)

UNESCO, (1998). Ejes Transversales Aprendizajes para la vida. Recuperado de: http://recursosbiblio.url.edu.gt/publicjlg/Lib/2015/url/06/los_eje.pdf 